\title{
Spin-Dependent Transport through SU(4) Kondo Dot in the Presence of Spin-Flip Processes
}

\author{
D. KRYCHOWSKI AND S. LIPIŃSKI \\ Institute of Molecular Physics, Polish Academy of Sciences, M. Smoluchowskiego 17, 60-179 Poznań, Poland \\ The spin-resolved current of carbon nanotube quantum dot coupled to ferromagnetic electrodes and influenced \\ by spin-flip scattering is studied in the Kondo regime by the equation of motion method. \\ PACS numbers: 73.22.-f, 73.23.-b, 73.63.Fg
}

\section{Introduction}

Carbon nanotube quantum dot (CNT-QD) is a unique system exhibiting exotic many-body spin-orbital Kondo effect [1].The long spin lifetimes of carbon nanotubes, well elaborated technology of their coupling to ferromagnetic electrodes [2] and relatively high Kondo temperatures [1] offer the prospect for using Kondo effect in spintronics. The aim of the present paper is to discuss the combined influence of ferromagnetic leads and spin-flip processes on spin-resolved transport in these systems.

\section{Model}

The low energy band structure of semiconducting carbon nanotubes is orbitally doubly degenerate. This degeneracy corresponds to clockwise and counterclockwise symmetry of the wrapping modes in CNTs [3]. In the present considerations we restrict to the single shell and the dot is modeled by double orbital Anderson Hamiltonian with equal intraorbital and the interorbital Coulomb interaction $(\mathcal{U})$ and spin flip scattering in the dot parameterized by $\mathcal{R}$ :

$$
\begin{aligned}
\mathcal{H} & =\sum_{k \alpha m \sigma} \epsilon_{k \alpha m \sigma} c_{k \alpha m \sigma}^{+} c_{k \alpha m \sigma}+\sum_{m \sigma} \epsilon_{m \sigma} d_{m \sigma}^{+} d_{m \sigma} \\
& +\sum_{k \alpha m \sigma} t_{\alpha}\left(c_{k \alpha m \sigma}^{+} d_{m \sigma}+\text { H.c. }\right)+\sum_{m} \mathcal{U} n_{m+} n_{m-} \\
& +\sum_{\sigma \sigma^{\prime}} \mathcal{U} n_{1 \sigma} n_{-1 \sigma^{\prime}}+\sum_{m} \mathcal{R}\left(d_{m+}^{+} d_{m-}+\text { H.c. }\right),
\end{aligned}
$$

where $m= \pm 1$ numbers the orbitals, the leads channels are labeled by $(m, \alpha), \alpha=\mathrm{L}, \mathrm{R}, \epsilon_{m \sigma}=\epsilon_{0}-e V_{\mathrm{g}}$ and $V_{\mathrm{g}}$ denotes gate voltage. We set $|e|=|g|=\left|\mu_{\mathrm{B}}\right|=\left|k_{\mathrm{B}}\right|=$ $|h|=1$. The spin polarization of the leads $\mathcal{P}=\mathcal{P}_{\alpha}$ is defined by spin-dependent densities of states $\left(\varrho_{\alpha \sigma}\right)$, $\mathcal{P}_{\alpha}=\left(\varrho_{\alpha+}-\varrho_{\alpha-}\right) /\left(\varrho_{\alpha+}+\varrho_{\alpha-}\right)$. Current flowing through CNT-QD in the $|m \sigma\rangle$ channel $\mathcal{I}_{m \sigma}=\left(\mathcal{I}_{\mathrm{L} m \sigma}-\mathcal{I}_{\mathrm{R} m \sigma}\right) / 2$ can be expressed in term of the lesser Green functions as follows [4]:

$$
\mathcal{I}_{\alpha m \sigma}=\sum_{k} t_{\alpha}\left[G_{m \sigma, k \alpha m \sigma}^{<}(t)-G_{k \alpha m \sigma, m \sigma}^{<}(t)\right] .
$$

The corresponding conductances are defined as $\mathcal{G}_{m \sigma}=$ $\mathrm{d} \mathcal{I}_{m \sigma} / \mathrm{d} V, \mathcal{G}_{\sigma}=\mathcal{G}_{1 \sigma}+\mathcal{G}_{-1 \sigma}$ and polarization of conductance $\mathrm{PC}=\left(\mathcal{G}_{+}-\mathcal{G}_{-}\right) /\left(\mathcal{G}_{+}+\mathcal{G}_{-}\right)$. The Green functions are found by the equation of motion method using the Lacroix decoupling, which preserves the Kondo correlations [5] and the lesser Green functions $G^{<}$are determined using the $\mathrm{Ng}$ ansatz [6], according to which the lesser self-energy $\Sigma^{<}$is proportional to the self-energy of the corresponding noninteracting system $\Sigma^{<}(\omega)=$ $\Lambda \Sigma^{0<}(\omega)$, and $\Lambda$ can be found by the Keldysh requirement $\Sigma^{\mathrm{P}}-\Sigma^{<}=\Sigma^{\mathrm{R}}-\Sigma^{\mathrm{A}}$, where $\Sigma^{\mathrm{R}(\mathrm{A})}$ are retarded (advanced) self-energies. The role of polarization of electrodes is twofold: it makes the tunneling induced broadenings spin dependent $\Gamma_{\alpha m \sigma}=2 \pi t_{\alpha}^{2} \varrho_{\alpha m \sigma}$, and it induces an effective exchange splitting $\left(\Delta_{\text {exch }}\right)$ via spin-dependent charge fluctuations. For brevity we do not cite here the explicit formula for $\Delta_{\text {exch }}$, this can be found e.g. in [7].

\section{Results and discussion}

We present numerical results for $\epsilon_{0}=-6$ and $\mathcal{U}=15$ ( $\Gamma=\sum_{\alpha m \sigma} \Gamma_{\alpha m \sigma}$ is taken as the energy unit). Figures $1 \mathrm{a}, \mathrm{b}$ present polarization of conductance of CNT-QD in the Kondo regime versus gate and bias voltages for different values of spin-flip scattering amplitudes. Figures 1c,d show generalized transmissions $\mathcal{T}^{\sigma \sigma}=4\left(\Gamma_{\mathrm{L}} G^{\mathrm{R}} \Lambda \Gamma_{\mathrm{R}} G^{\mathrm{A}}\right)[8,9]$, which incorporate part of the nonequilibrium effects of the Coulomb interaction. Polarization of electrodes breaks the spin degeneracy leading to a crossover from spin-orbital SU(4) to coupled orbital SU(2) Kondo effect. For $\mathcal{R}=0$ the three peak structure of transmission is observed $\left(E \approx T_{\mathrm{K}}\right.$, $\left.E \approx T_{\mathrm{K}} \pm \Delta_{\text {exch }}\right)$ and for $\mathcal{R} \neq 0$ the satellites reflecting spin and spin-orbital fluctuations further split and locate around $E \approx T_{\mathrm{K}} \pm\left(\Delta_{\mathrm{exch}} \pm 2 \mathcal{R}\right)$, where $T_{\mathrm{K}}$ denotes polarization and spin-flip perturbed Kondo temperature. For small values of exchange splitting all the peaks are 

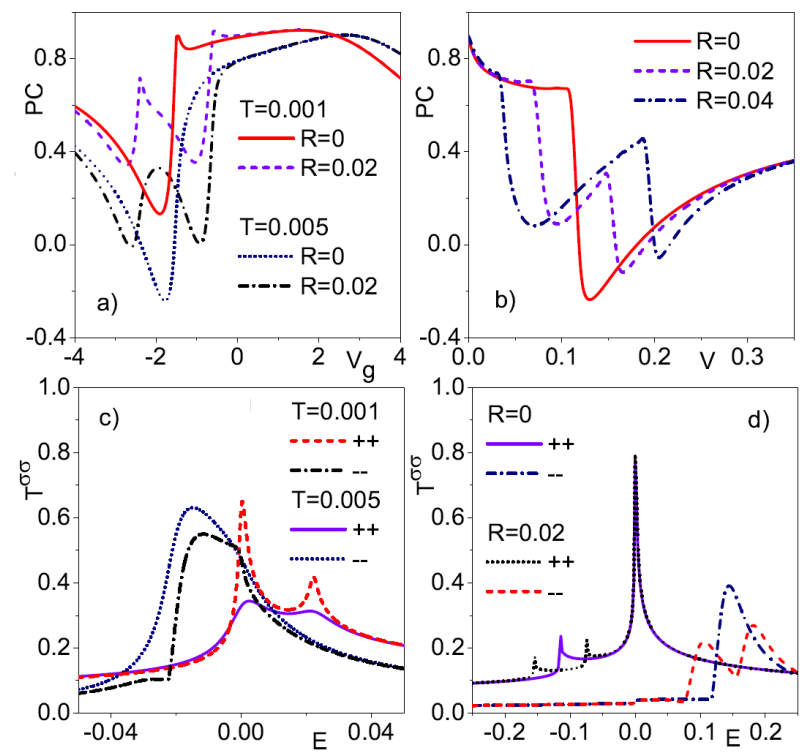

Fig. 1. Polarization of conductance of CNT-QD coupled to ferromagnetic electrodes $(\mathcal{P}=0.6)$ in the Kondo regime for different values of spin-flip amplitude (a) vs. gate voltage, $(\mathrm{b})$ vs. bias voltage $\left(V_{\mathrm{g}}=1, T=0.001\right)$. Generalized spin resolved transmissions of CNT-QD for gate voltage $(\mathrm{c}) V_{\mathrm{g}}=-2(\mathcal{R}=0)$, (d) $V_{\mathrm{g}}=1$ $(T=0.001)$.

located close to $E_{\mathrm{F}}$ (Fig. $\left.1 \mathrm{c}, V_{\mathrm{g}} \approx-2, \Delta_{\mathrm{exch}} \approx 0.02\right)$. Location near $E_{\mathrm{F}}$ explains strong impact of spin-flip scattering or temperature on linear $\mathrm{PC}$ in this region. The ratio of spin resolved linear transmissions is easily disturbed in this case. As it is seen in Fig. 1a in this range suppression $(T=0.001)$ or even a change of sign of linear
PC $(T=0.005)$ can result. For gate voltages away from $V_{\mathrm{g}}=-2\left(\left|\Delta_{\text {exch }}\right|>T_{\mathrm{K}}\right)$ linear $\mathrm{PC}$ reaches high values and is more robust against spin flips, which is especially visible for positive $V_{\mathrm{g}}$ (Fig. 1a). This is a consequence of strong dominance of majority spin linear transmission around the Fermi energy in this range (Fig. 1d, $V_{\mathrm{g}}=-1$, $\Delta_{\text {exch }}=-0.12$ ). Figure $1 \mathrm{~b}$ shows bias dependence of PC. Local minima of $\mathrm{PC}(V)$ are observed when bias voltage $V$ sweeps across new resonances (Fig. 1d).

\section{References}

[1] P. Jarillo-Herrero, J. Kong, H.S.J. Van der Zant, C. Dekker, L.P. Kouvenhoven, S. De Franceschi, $\mathrm{Na}$ ture 434, 484 (2005).

[2] A. Cottet, T. Kontos, S. Sahoo, H.T. Man, M.-S. Choi, W. Belzig, C. Bruder, A.F. Marpugo, C. Schoenberger, Semicond. Sci. Technol. 21, S78 (2006).

[3] W. Liang, M. Bockrath, H. Park, Phys. Rev. Lett. 88, 126801 (2002).

[4] H. Haug, A.-P. Jauho, Quantum Kinetics in Transport and Optics of Semiconductors, Springer, Berlin 1998.

[5] C. Lacroix, J. Phys. F 11, 2389 (1998).

[6] T.K. Ng, Phys. Rev. Lett. 76, 487 (1996).

[7] J. Martinek, M. Sindel, L. Borda, J. Barnaś, R. Bulla, J. Konig, G. Schon, S. Maekawa, J. von Delft, Phys. Rev. B 72, 121302(R) (2005).

[8] T.-F. Fang, S.-J. Wang, J. Phys., Condens. Matter. 19, 026204 (2007).

[9] S. Lipiński, D. Krychowski, Phys. Rev. B 81, 115327 (2010). 